\title{
Fragmentation and dynamical collapse of the starless high-mass star-forming region IRDC 18310-4 $\star, \star \star$
}

\author{
H. Beuther ${ }^{1}$, H. Linz ${ }^{1}$, J. Tackenberg, Th. Henning ${ }^{1}$, O. Krause ${ }^{1}$, S. Ragan ${ }^{1}$, M. Nielbock ${ }^{1}$, R. Launhardt ${ }^{1}$, S. Bihr ${ }^{1}$, \\ A. Schmiedeke ${ }^{1,2}$, R. Smith $^{3}$, and T. Sakai ${ }^{4}$ \\ 1 Max-Planck-Institute for Astronomy, Königstuhl 17, 69117 Heidelberg, Germany \\ e-mail: name@mpia.de \\ 2 University of Cologne, Zülpicher Strasse 77, 50937 Köln, Germany \\ ${ }^{3}$ Institute for Theoretical Astrophysics, Univ. of Heidelberg, Albert-Ueberle-Str. 2, 69120 Heidelberg, Germany \\ ${ }^{4}$ Institute of Astronomy, The University of Tokyo, Osawa, Mitaka, 181-0015 Tokyo, Japan
}

Received 1 October 2012 / Accepted 4 April 2013

\begin{abstract}
Context. Because of their short evolutionary time-scales, the earliest stages of high-mass star formation prior to the existence of any embedded heating source have barely been characterized until today.

Aims. We study the fragmentation and dynamical properties of a massive starless gas clump at the onset of high-mass star formation. Methods. Based on Herschel continuum data we identify a massive gas clump that remains far-infrared dark up to $100 \mu \mathrm{m}$ wavelengths. The fragmentation and dynamical properties are investigated by means of Plateau de Bure Interferometer and Nobeyama $45 \mathrm{~m}$ single-dish spectral line and continuum observations.

Results. The massive gas reservoir (between $\sim 800$ and $\sim 1600 M_{\odot}$, depending on the assumed dust properties) fragments at spatial scales of $\sim 18000 \mathrm{AU}$ in four cores. Comparing the spatial extent of this high-mass region with intermediate- to low-mass starless cores from the literature, we find that linear sizes do not vary significantly over the whole mass regime. However, the high-mass regions squeeze much more gas into these similar volumes and hence have orders of magnitude larger densities. The fragmentation properties of the presented low-to high-mass regions are consistent with gravitational instable Jeans fragmentation. Furthermore, we find multiple velocity components associated with the resolved cores. Recent radiative transfer hydrodynamic simulations of the dynamic collapse of massive gas clumps also result in multiple velocity components along the line of sight because of the clumpy structure of the regions. This result is supported by a ratio between viral and total gas mass for the whole region $<1$.

Conclusions. This apparently still starless high-mass gas clump exhibits clear signatures of early fragmentation and dynamic collapse prior to the formation of an embedded heating source. A comparison with regions of lower mass reveals that the linear size of star-forming regions does not necessarily have to vary much for different masses, however, the mass reservoirs and gas densities are orders of magnitude enhanced for high-mass regions compared to their lower-mass siblings.
\end{abstract}

Key words. stars: early-type - stars: formation - stars: individual: IRDC18310-4 - stars: massive - ISM: kinematics and dynamics ISM: clouds

\section{Introduction}

Independent of the various formation scenarios for high-mass stars that are discussed extensively in the literature (e.g., Zinnecker \& Yorke 2007; Beuther et al. 2007), the initial conditions required to allow high-mass star formation at all are still poorly characterized. The initial debate even ranged around the question whether high-mass starless gas clumps should exist at all, or whether the collapse of massive gas clumps starts immediately without any clear starless phase in the high-mass regime (e.g., Motte et al. 2007). Recent studies indicate that the time where massive gas clumps exist without embedded star formation is relatively short (on the order of $50000 \mathrm{yrs}$ ), but nevertheless, high-mass starless gas clumps do exist (e.g., Russeil et al. 2010; Tackenberg et al. 2012).

* Based on observations carried out with the IRAM Plateau de Bure Interferometer. IRAM is supported by INSU/CNRS (France), MPG (Germany) and IGN (Spain).

$\star \star$ The data are available at the CDS via anonymous ftp to cdsarc.u-strasbg.fr (130.79.128.5) or via

http://cdsarc.u-strasbg.fr/viz-bin/qcat?]/A+A/553/A115
Current questions in that field are: Are high-mass gas clumps dominated by a single fragment or do we witness already strong fragmentation during earliest evolutionary stages (e.g., Bontemps et al. 2010)? What are the kinematic properties of the gas? Are the clumps sub- or super-virial? Do we see streaming motions indicative of turbulent flows (e.g., Bergin et al. 2004; Vázquez-Semadeni et al. 2006; Heitsch et al. 2008; Banerjee et al. 2009)?

To address these questions high spatial resolution is mandatory. Furthermore, to study the dense gas, dust continuum as well as spectral line observations are required. Therefore we tackle these questions via a dedicated study of the genuine high-mass starless gas clump IRDC 18310-4 that was first identified by Sridharan et al. (2005). Their early study based on $1.2 \mathrm{~mm}$ continuum emission, $8 \mu \mathrm{m}$ extinction and $\mathrm{NH}_{3}$ emission revealed an infrared dark gas clump with a mass of $\sim 840 M_{\odot}$, a temperature of $\sim 18 \mathrm{~K}$, and a $\mathrm{NH}_{3}(1,1)$ line-width measured with the Effelsberg $100 \mathrm{~m}$ telescope with $40^{\prime \prime}$ spatial resolution of $\sim 1.7 \mathrm{~km} \mathrm{~s}^{-1}$. The kinematic distance of the source is $\sim 4.9 \pm 0.26 \mathrm{kpc}$ (Ragan et al. 2012a, recalculated following Reid et al. 2009) and its velocity of rest $v_{\text {lsr }} \sim 86.5 \mathrm{~km} \mathrm{~s}^{-1}$ (Sridharan et al. 2005). 
The target region is at a projected separation of $\sim 3.4^{\prime}$ (at the given distance corresponding to $\sim 5.1 \mathrm{pc}$ ) from the luminous high-mass protostellar object (HMPO) IRAS 18310-0825 (Sridharan et al. 2002; Beuther et al. 2002) but otherwise in no outstanding part of our Galaxy. This region is part of the Herschel Earliest Phases of Star Formation (EPoS) key project that studies a large sample of high- and low-mass star-forming regions at the earliest evolutionary stages (Ragan et al. 2012a; Launhardt et al. 2013; Henning et al. 2010; Linz et al. 2010; Beuther et al. 2010, 2012b; Stutz et al. 2010).

Except for these characterizations, little additional information exist for that region. Here, we present Herschel far-infrared continuum observations as well as single-dish and interferometrically obtained $\mathrm{N}_{2} \mathrm{H}^{+}(1-0)$ observations from the Nobeyama $45 \mathrm{~m}$ telescope and the Plateau de Bure Interferometer (PdBI). The latter data also allow us to image high-spatial-resolution $3 \mathrm{~mm}$ continuum data.

\section{Observations}

\subsection{Herschel}

The cloud complex with a size of $\sim 6^{\prime} \times 6^{\prime}$ was observed with PACS (Poglitsch et al. 2010) on Herschel (Pilbratt et al. 2010) on April 19th, 2011 (PACS OBS ID 134221906[0-3]). Scan maps in two orthogonal directions with scan leg lengths of $\sim 10^{\prime}$ were obtained with the medium scan speed of $20^{\prime \prime} / \mathrm{s}$. The raw data have been reduced up to level-1 with the HIPE software (Poglitsch et al. 2010; Ott 2010), version 6.0, build 1932. Besides the standard steps, we applied a 2nd level deglitching, in order to remove bad data values from a given pixel map by $\sigma$-clipping the flux values which contribute to each pixel. We used the time-ordered option and applied a $25 \sigma$ threshold. The final level-2 maps were processed using Scanamorphos version 8 (Roussel 2012). Since the field of view contains bright emission on scales larger than the map, we applied the "galactic" option and included the nonzero-acceleration telescope turn-around data. The flux correction factors provided by the PACS ICC team were applied. The $70 \mu \mathrm{m}$ extinction feature and the PdBI continuum and line data exhibited a small offset of approximately $3^{\prime \prime}$ in RA and $1^{\prime \prime}$ in Dec. Although the interferometer positional accuracy is usually superior because of the extremely well-known positions of the reference quasars, we double-checked the $70 \mu \mathrm{m}$ PACS data via a comparison to the Spitzer MIPSGAL $24 \mu \mathrm{m}$ survey which has also a very high spatial accuracy (Carey et al. 2009). Again, the same offset between MIPSGAL $24 \mu \mathrm{m}$ and Herschel $70 \mu \mathrm{m}$ was found. These Herschel data were taken at still an early Herschel time, and positional offsets on this order are known during that time. Therefore, we shifted the $70 \mu \mathrm{m}$ map by this little offset in the high-resolution overlays from Fig. 2 onwards. The spatial resolution of the 70, 100 and $160 \mu \mathrm{m}$ data is $5.6^{\prime \prime}, 6.8^{\prime \prime}$ and $11.4^{\prime \prime}$, respectively.

Maps at 250, 350, and $500 \mu \mathrm{m}$ were obtained with SPIRE (Griffin et al. 2010) on March 11th, 2010 (SPIRE OBS ID 1342192067). Two times two $20^{\prime}$ scan legs were used to cover the source. The data were processed up to level-1 within HIPE, developer build 5.0, branch 1892, calibration tree 5.1 using the standard photometer script (POF5_pipeline.py, dated 2.3.2010) provided by the SPIRE ICC team. The resulting level1 maps have been further reduced using Scanamorphos, version 9 (patched, dated 08.03.2011). This version included again the essential de-striping for maps with less than 3 scan legs per scan. In addition, we used the "galactic" option and included the non-zero-acceleration telescope turn-around data. Corrections for the transition from point sources to extended sources were applied according to the SPIRE Manual. The spatial resolution of the 250,350 and $500 \mu \mathrm{m}$ data is $18.1^{\prime \prime}, 24.9^{\prime \prime}$ and $36.6^{\prime \prime}$, respectively.

\subsection{Plateau de Bure Interferometer}

We observed IRDC 18310-4 with the PdBI during five nights in October and November 2009 at $93 \mathrm{GHz}$ in the C and D configurations covering projected baselines between approximately 13 and $175 \mathrm{~m}$. The observations were conducted in a track-sharing mode together with the IRDC 18454-1 in the neighborhood of W43 (Beuther et al. 2012b). The $3 \mathrm{~mm}$ receivers were tuned to $92.835 \mathrm{GHz}$ in the lower sideband covering the $\mathrm{N}_{2} \mathrm{H}^{+}(1-0)$ as well as the $3.23 \mathrm{~mm}$ continuum emission. At the given frequency, the primary beam of the PdBI is $\sim 54^{\prime \prime}$. For continuum measurements we placed six $320 \mathrm{MHz}$ correlator units in the band, the spectral lines were excluded in averaging the units to produce the final continuum image. Temporal fluctuations of amplitude and phase were calibrated with frequent observations of the quasars $1827+062$ and 1829-106. The amplitude scale was derived from measurements of MWC 349 and 3c454.3. We estimate the final flux accuracy to be correct to within $\sim 15 \%$. The phase reference center is RA (J2000.0) 18:33:39.468 and Dec (J2000.0) -08:21:07.65, and the velocity of rest $v_{\mathrm{lsr}}$ is $86.5 \mathrm{~km} \mathrm{~s}^{-1}$ for the two velocity components, respectively. The data were imaged with a robust weighting scheme close to uniform weighting. The synthesized beam of the line and continuum data is $4.3^{\prime \prime} \times 3.0^{\prime \prime}$ with a PA of 14 degrees. The $3 \sigma$ continuum $\mathrm{rms}$ is $0.24 \mathrm{mJy}_{\text {beam }}{ }^{-1}$. The $3 \sigma \mathrm{rms}$ of the $\mathrm{N}_{2} \mathrm{H}^{+}(1-0)$ data measured from an emission-free channel with a spectral resolution of $0.2 \mathrm{~km} \mathrm{~s}^{-1}$ is $21 \mathrm{mJy}$ beam $^{-1}$. Data calibration and imaging was performed with the GILDAS software package ${ }^{1}$.

\subsection{Nobeyama $45 \mathrm{~m}$ telescope}

The $\mathrm{N}_{2} \mathrm{H}^{+}$data has been observed using the BEARS receiver at the NRO $45 \mathrm{~m}$ telescope in Nobeyama, Japan, in April 2010. The receiver has been tuned to $93.17346 \mathrm{MHz}$, covering the full hyperfine structure of the $\mathrm{N}_{2} \mathrm{H}^{+}(1-0)$ transition with average system temperatures of $T_{\text {sys }} \sim 200 \mathrm{~K}$. At this frequency the telescope beam is $18.2^{\prime \prime}$ and the observing mode provides a spectral resolution of $0.2 \mathrm{~km} \mathrm{~s}^{-1}$ at a bandwidth of $32 \mathrm{MHz}$. The software package nostar (Sawada et al. 2008) was used for the data reduction, sampling the data to a pixel size of $7^{\prime \prime}$ with a spheroidal convolution and smoothing the spectral resolution to $0.5 \mathrm{~km} \mathrm{~s}^{-1}$. The rms of the final map is $\sim 0.11 \mathrm{~K}$ per $0.5 \mathrm{~km} \mathrm{~s}^{-1}$ wide channel. Strong winds hampered the pointing for part of the observations, contributing to spatial uncertainties.

\subsection{Merging of $P d B I$ and Nobeyama data}

To combine the $\mathrm{N}_{2} \mathrm{H}^{+}(1-0)$ from the Nobeyama $45 \mathrm{~m}$ with the $\mathrm{PdBI}$ data, the Nobeyama data were converted into units of Jy, and then the combination was done within the GILDAS software package by the task UVSHORT. The synthesized beam of the combined dataset is $5.1^{\prime \prime} \times 3.2^{\prime \prime}$ with a PA of 8 degrees. The $3 \sigma$ rms of the merged $\mathrm{N}_{2} \mathrm{H}^{+}(1-0)$ data measured from an emission-free channel with a spectral resolution of $0.2 \mathrm{~km} \mathrm{~s}^{-1}$ is $54 \mathrm{mJy}^{\text {beam }}{ }^{-1}$.

http://www.iram.fr/IRAMFR/GILDAS 


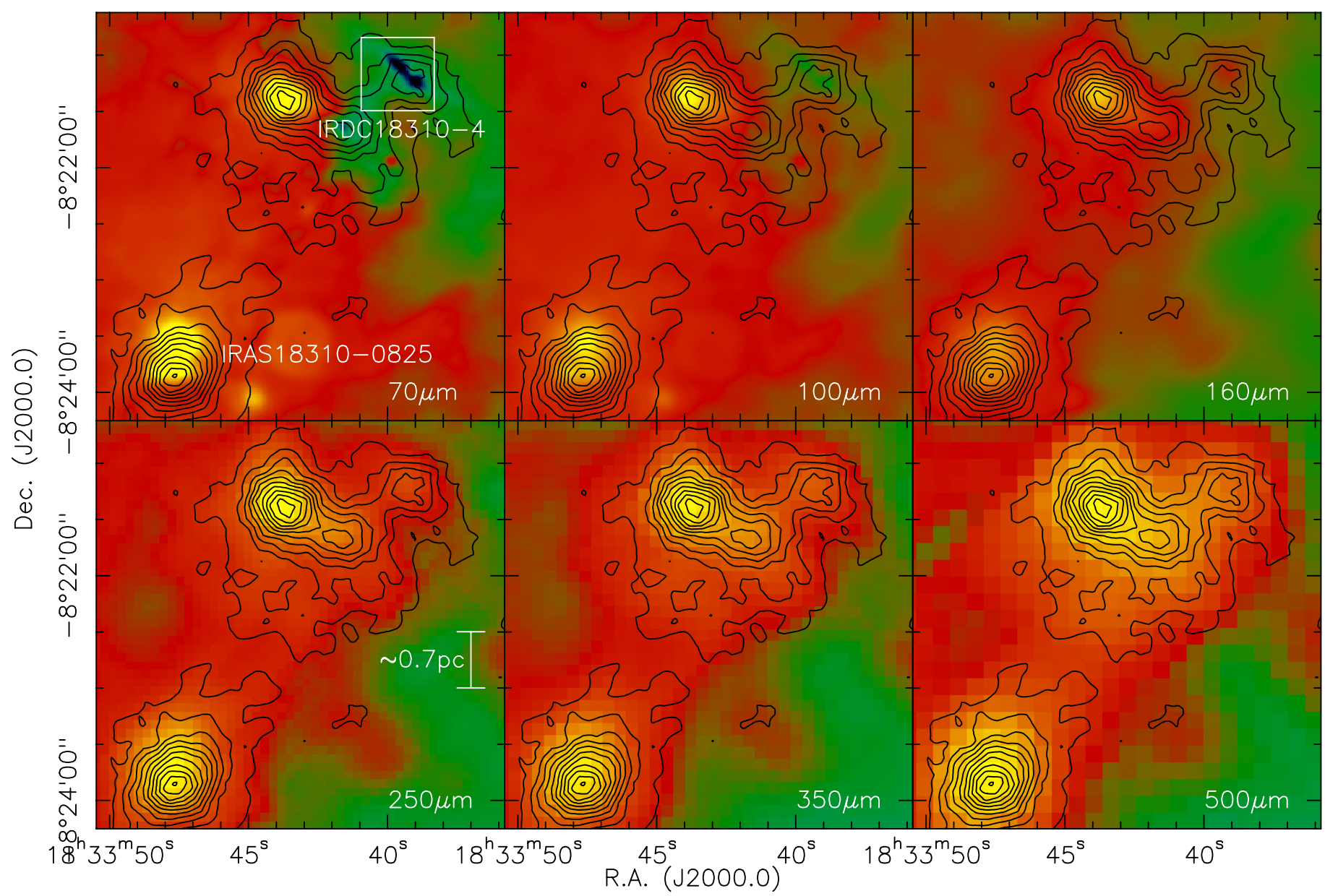

Fig. 1. Compilation of the continuum data from $70 \mu \mathrm{m}$ to $500 \mu \mathrm{m}$ wavelength in color-scale as labeled in each panel. The scale is chosen in each image individually to highlight the most important features. Contour levels of the $870 \mu \mathrm{m}$ data start at the $3 \sigma$ levels of $0.189 \mathrm{mJy}^{\mathrm{beam}}{ }^{-1}$ and continue in in $3 \sigma$ steps. The white small box in the top-left panel marks the smaller region discussed in the remaining figures. A scale-bar is shown in the bottom-left panel.

\subsection{APEX and the IRAM $30 \mathrm{~m}$ continuum data}

The $1.2 \mathrm{~mm}$ continuum data were first presented in Beuther et al. (2002) and the APEX $870 \mu \mathrm{m}$ data are part of the ATLASGAL survey of the Galactic plane (Schuller et al. 2009). Beam sizes of the two datasets are $10.5^{\prime \prime}$ and $19.2^{\prime \prime}$, respectively. The $1 \sigma \mathrm{rms}$ values are 12 and $63 \mathrm{mJy}$ beam $^{-1}$, respectively.

\section{Results}

\subsection{Continuum emission}

Figure 1 presents an overview of the far-infrared to submm continuum data obtained toward the whole cloud complex with Herschel and APEX. While the IRAS source and an additional far-infrared source in the north are bright at all presented wavelengths, our target region IRDC 18310-4 shows considerable structure changes between $70 \mu \mathrm{m}$ and $870 \mu \mathrm{m}$. While it is a strong emission source at long wavelengths, the source is only depicted as an absorption shadow at 70 and $100 \mu \mathrm{m}$. While we cannot exclude that some ongoing low-mass star formation may exist below our detection threshold, non-detections at farinfrared wavelengths are among the best indicators for genuine high-mass starless gas clumps.

The longest wavelengths $1.2 \mathrm{~mm}$ continuum data with a spatial resolution of 10.5" first presented in Beuther et al. (2002) are best suited to re-estimate the gas mass and column densities of IRDC 18310-4. Peak and integrated $1.2 \mathrm{~mm}$ fluxes are $132 \mathrm{mJy}$ beam $^{-1}$ and $717 \mathrm{mJy}$ for that region, respectively. Assuming optically thin thermal emission from dust, a gas-todust mass ratio of 186 (Jenkins 2004; Draine et al. 2007) and a temperature of $18 \mathrm{~K}$ derived from $\mathrm{NH}_{3}$ observations (Sridharan et al. 2005) as well as the Herschel continuum data (see below), we can estimate masses and column densities for different dust properties. Using standard ISM dust properties (Mathis et al. 1977, MRN1977, $\left.\left(\kappa_{1.2 \mathrm{~mm}} \sim 0.4 \mathrm{~cm}^{2} \mathrm{~g}^{-1}\right)\right)$, the clump mass and peak column density are $\sim 1600 M_{\odot}$ and $2.5 \times 10^{23} \mathrm{~cm}^{-2}$, respectively. If we use instead the dust properties discussed in Ossenkopf \& Henning (1994) for thin ice mantles at densities of $10^{5} \mathrm{~cm}^{-3}\left(\mathrm{OH} 94, \kappa_{1.2 \mathrm{~mm}} \sim 0.8 \mathrm{~cm}^{2} \mathrm{~g}^{-1}\right)$, the corresponding values for mass and column density are $\sim 800 M_{\odot}$ and $1.3 \times 10^{23} \mathrm{~cm}^{-2}$, respectively. These two estimates can be considered as brackets around the real gas masses and column densities of that region.

Assuming a standard initial mass function (e.g., Kroupa 2001) and a relatively high star formation efficiency of $\sim 30 \%$ which should be appropriate for such dense gas clumps (e.g., Alves et al. 2007), one needs approximately $1000 M_{\odot}$ initial gas mass to form a cluster with at least one $20 M_{\odot}$ star in the end. Given the mass ranges estimated above, IRDC 18310-4 should be capable of high-mass star formation. Considering column density thresholds, Tackenberg et al. (2012) recently estimated that the initial gas column density for the Orion Nebula Cluster should have been approximately $1.8 \times 10^{23} \mathrm{~cm}^{-2}$, which roughly agrees with the theoretical column density threshold 
Table 1. Core parameters from the PdBI $3.2 \mathrm{~mm}$ data.

\begin{tabular}{lcc|cc|cc|cc|cc|cc|c}
\hline \hline$\#$ & $\begin{array}{c}\text { RA } \\
(\mathrm{J} 2000.0)\end{array}$ & $\begin{array}{c}\text { Dec } \\
(\mathrm{J} 2000.0)\end{array}$ & $\begin{array}{c}S_{\text {peak }} \\
\left(\frac{\mathrm{mJy}}{\mathrm{beam}}\right)\end{array}$ & $\begin{array}{c}S \\
(\mathrm{mJy})\end{array}$ & $\begin{array}{c}N_{\mathrm{H}_{2}} \\
\left(\frac{10^{23}}{\mathrm{~cm}^{2}}\right)\end{array}$ & $\begin{array}{c}M \\
\left(M_{\odot}\right)\end{array}$ & $\begin{array}{c}N_{\mathrm{H}_{2}} \\
\left(\frac{10^{23}}{\mathrm{~cm}^{2}}\right)\end{array}$ & $\begin{array}{c}M \\
\left(M_{\odot}\right)\end{array}$ & $\begin{array}{c}\Delta v_{1} \\
\left(\mathrm{~km} \mathrm{~s}^{-1}\right)\end{array}$ & $\begin{array}{c}M_{\mathrm{vir}} \\
\rho \propto \frac{1}{r},\left(M_{\odot}\right) \\
v_{1}\end{array}$ & $\begin{array}{c}M_{\text {vir }} \\
v_{2}\end{array}$ & $\begin{array}{c}M_{\mathrm{vir}} \\
\rho \propto \frac{1}{r^{2}}, \\
v_{1}\end{array}$ & $\begin{array}{c}M_{\text {vir }} \\
\left(M_{\odot}\right) \\
v_{2}\end{array}$ \\
\hline $\mathrm{mm} 1$ & $18: 33: 39.472$ & $-08: 21: 09.96$ & 0.90 & 1.2 & 6.1 & 107 & 2.0 & 36 & 2.4 & 0.6 & 48 & 3 & 32 \\
$\mathrm{~mm} 2$ & $18: 33: 39.277$ & $-08: 21: 09.83$ & 0.75 & 0.9 & 5.1 & 81 & 1.7 & 27 & 2.4 & 0.8 & 48 & 5 & 32 \\
$\mathrm{~mm} 3$ & $18: 33: 39.334$ & $-08: 21: 16.74$ & 0.91 & 1.2 & 6.2 & 107 & 2.1 & 36 & 2.0 & 0.6 & 33 & 3 & 22 \\
$\mathrm{~mm} 4$ & $18: 33: 40.013$ & $-08: 21: 04.84$ & 0.47 & 0.6 & 3.2 & 54 & 1.1 & 18 & - & - & & \\
\hline
\end{tabular}

Notes. MRN1977 Mathis et al. (1977); OH94 Ossenkopf \& Henning 1994; $v 1$ and $v 2$ correspond to the fitted peak velocities of the two components in Fig. 6.

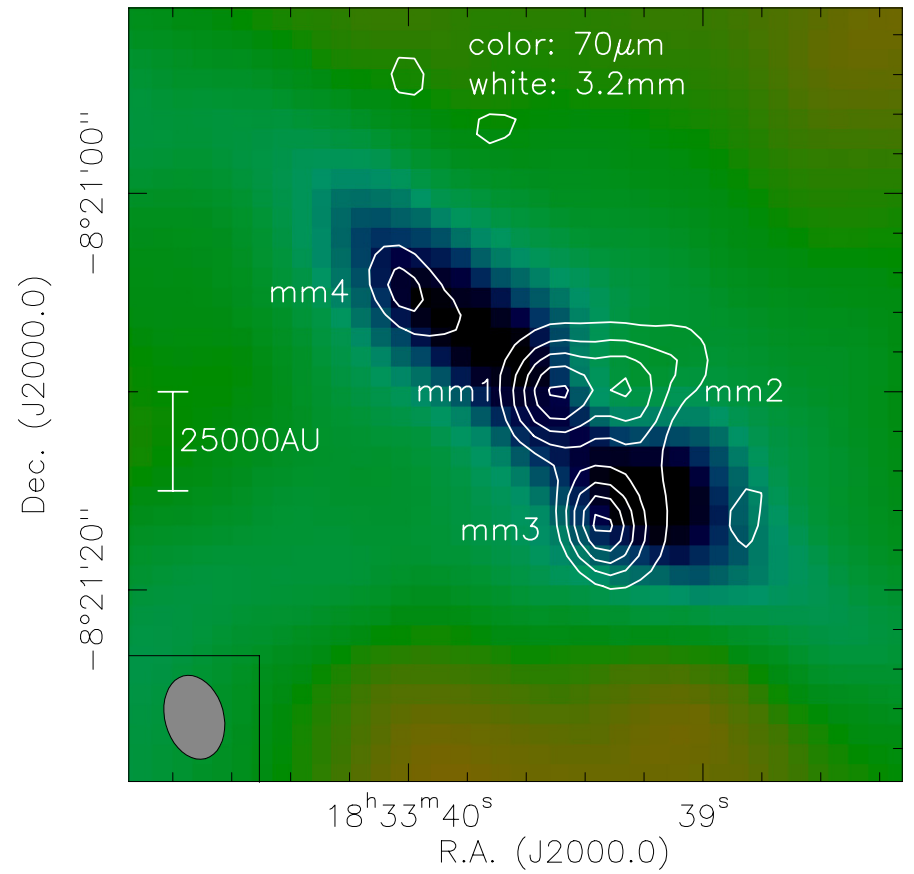

Fig. 2. Zoom into the central IRDC 18310-4. The color-scale shows the $70 \mu \mathrm{m}$ extinction feature, and the contours present the $3.2 \mathrm{~mm}$ $\mathrm{PdBI}$ continuum emission starting at $3 \sigma$ and continuing in $2 \sigma$ steps $\left(1 \sigma=0.08 \mathrm{mJy} \mathrm{beam}^{-1}\right)$. The mm-labels, a synthesized beam and a scale-bar are shown as well. The phase reference center is close to $\mathrm{mm} 1$ at RA (J2000.0) 18:33:39.468 and Dec (J2000.0) - 08:21:07.65, and the primary beam of the observations with $\sim 54^{\prime \prime}$ is larger than the shown image size.

from Krumholz \& McKee (2008). Comparing this to the peak column density derived from the $1.2 \mathrm{~mm}$ data above, it also indicates that this region may form high-mass stars in the future. Commerçon et al. (2011) recently pointed out that not only high column density and heating can prevent fragmentation but that the magnetic field may be as important.

Figure 2 presents a zoom toward our central target region IRDC 18310-4. With the PdBI $3.2 \mathrm{~mm}$ continuum data, we can identify four sub-sources ( $\mathrm{mm} 1$ to $\mathrm{mm} 4$, in the following also called cores, in contrast to the larger-scale environment labeled clump) above a $5 \sigma$ threshold. While we detect a few more $3 \sigma$ features, for the following analysis we only consider the $5 \sigma$ detections $\mathrm{mm} 1$ to $\mathrm{mm} 4$. The cores are marginally resolved, and the source separation between $\mathrm{mm} 1$ to $\mathrm{mm} 3$ is done "by eye" at the lowest flux decrements between the cores. Outer edges are chosen at the $3 \sigma$ level. Peak and integrated fluxes for these four cores are presented in Table 1. Using the dust emission again to estimate gas masses and column densities
Table 2. Projected nearest neighbor separations.

\begin{tabular}{ccc}
\hline \hline & $\left({ }^{\prime \prime}\right)$ & $(\mathrm{AU})$ \\
\hline $\mathrm{mm} 1-\mathrm{mm} 2$ & 2.9 & 14200 \\
$\mathrm{~mm} 1-\mathrm{mm} 3$ & 7.1 & 34900 \\
$\mathrm{~mm} 1-\mathrm{mm} 4$ & 9.5 & 46500 \\
\hline
\end{tabular}

with the same assumptions outlined above (MRN1977, $\kappa_{3.2 \mathrm{~mm}} \sim$

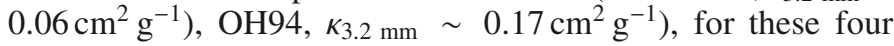
cores we find masses between 18 and $107 M_{\odot}$ and peak column densities between $1.1 \times 10^{23}$ and $6.1 \times 10^{23} \mathrm{~cm}^{-2}$, respectively (Table 1). While we obviously filter out a significant fraction of the flux with the interferometer $(\sim 73 \%)$, this region still hosts relatively massive cores within a high-mass gas clump reservoir.

While the locations of $\mathrm{mm} 1, \mathrm{~mm} 3$ and $\mathrm{mm} 4$ are largely associated with the $70 \mu \mathrm{m}$ extinction feature (although $\mathrm{mm} 1$ and $\mathrm{mm} 3$ are both shifted a bit to the edge), it is surprising that $\mathrm{mm} 2$ lies at the edge of the dark extinction feature at a narrowing of the extinction. Although we cannot identify a clear and isolated $70 \mu \mathrm{m}$ or shorter wavelength point source at this position, the slight increase of $70 \mu \mathrm{m}$ emission toward the $\mathrm{mm} 2$ peak is consistent with a very young embedded object that just started to heat up the environment. In this picture, it may be that $\mathrm{mm} 2$ is in a little more advanced evolutionary stage compared to the other $\mathrm{mm}$ peak positions.

Another characteristic of the fragmentation of the gas clump is the projected nearest neighbor separation which is listed in Table 2 . Since the beam is elongated in approximately northsouth direction, we can resolve in east-west direction a separation between $\mathrm{mm} 1$ and $\mathrm{mm} 2$ of about $\sim 2.9^{\prime \prime}$, which corresponds at the given distance of $4.9 \mathrm{kpc}$ to a projected linear separation of of $\sim 14200 \mathrm{AU}$. The projected nearest neighbor separations to the other cores is even larger, between $\sim 35000$ and $\sim 47000 \mathrm{AU}$ (Table 2). In comparison to these values, the approximate Jeanslength where gas clumps should fragment into sub-structures at approximate temperatures and densities of $18 \mathrm{~K}$ and $10^{5} \mathrm{~cm}^{-3}$ is $\sim 17000$ AU. Hence, we see fragmentation above and below this approximate Jeans-length which is reasonable since gas clumps have a density structure and therefore not one unique fragmentation scale.

We also use the Herschel data to derive the temperature structure of the region fitting the SEDs pixel by pixel (see also Stutz et al. 2010 for details of the fitting). We excluded the $500 \mu \mathrm{m}$ data to achieve a higher spatial resolution for the temperature map of $24.9^{\prime \prime}$, corresponding to the beam of the Herschel $350 \mu \mathrm{m}$ data. Figure 3 presents the results and we see a clear drop of the far-infrared dust temperature from the outside to the center of our region where temperatures around $18 \mathrm{~K}$ are measured. This is very similar to the gas temperature derived toward 


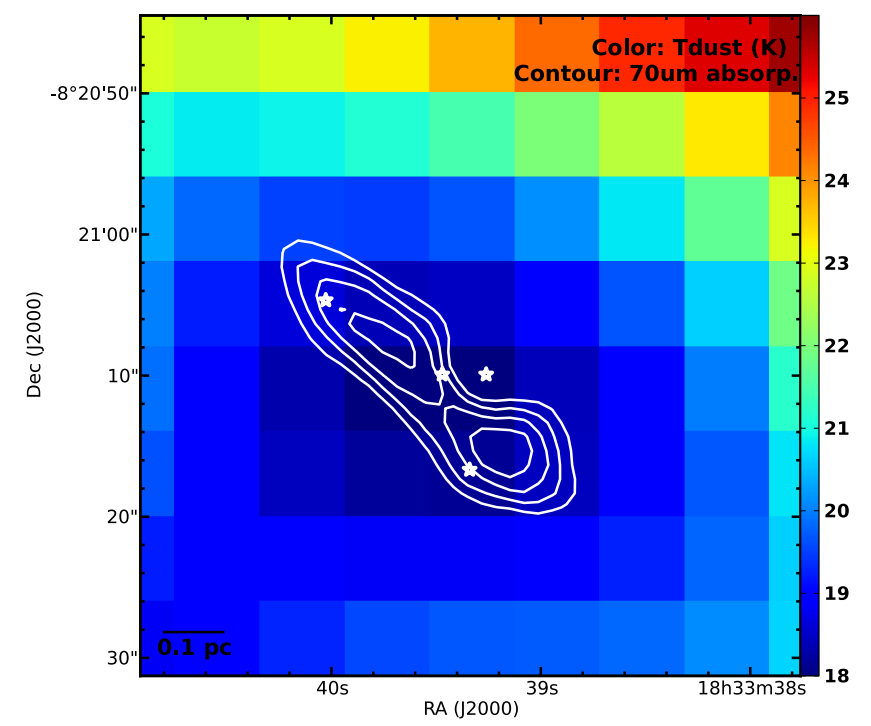

Fig. 3. The color-scale presents the dust temperature map derived from the Herschel far-infrared continuum data. Contours outline the structure of the $70 \mu \mathrm{m}$ extinction, and the stars mark the positions of the four mmsources from Fig. 2.

that region by $\mathrm{NH}_{3}$ observations (Sridharan et al. 2005), and it is further evidence that no internal heating source exists so far.

\subsection{Spectral line emission}

To study the kinematics of the gas, Fig. 4 presents the $\mathrm{N}_{2} \mathrm{H}^{+}(1-0)$ data (critical density at $20 \mathrm{~K}$ is $1.6 \times 10^{5} \mathrm{~cm}^{-3}$ ) of the region observed by Tackenberg et al. (2013). Although the $\mathrm{N}_{2} \mathrm{H}^{+}$emission toward IRDC 18310-4 is a bit weaker than toward the HMPO IRAS 18310-0825, we nevertheless see a clear $\mathrm{N}_{2} \mathrm{H}^{+}$peak toward our target region. Zooming into IRDC 18310-4, the top panel of Fig. 4 shows a 1st moment map (intensity-weighted peak velocity) extracted from these data using only the main hyperfine structure peak (see also Fig. 5). This figure shows that the overall velocity structure of the region is relatively uniform with no pronounced velocity structure and only weak gradients across the whole region $\left(<1 \mathrm{~km} \mathrm{~s}^{-1}\right.$ over $\sim 1$ pc extent).

Figure 5 shows a spectrum extracted toward the peak position. Observing the whole $\mathrm{N}_{2} \mathrm{H}^{+}(1-0)$ hyperfine structure simultaneously allows us to fit the optical depth and by that derive accurate spectral line parameters. For the single-dish spectrum toward the peak position, we find a line-width of $2.7 \mathrm{~km} \mathrm{~s}^{-1}$ at a velocity of $86.1 \mathrm{~km} \mathrm{~s}^{-1}$. The fitted peak velocity is very close to that measured previously in $\mathrm{NH}_{3}\left(86.5 \mathrm{~km} \mathrm{~s}^{-1}\right.$, Sridharan et al. 2005). Following MacLaren et al. (1988), we can estimate a virial mass assuming the FWHM/2 of the Nobeyama beam as the radius of the core. Employing possible density structures of $1 / r$ and $1 / r^{2}$ as border conditions, the virial mass of this region is between 305 and $202 M_{\odot}$, respectively. Compared to the total gas masses derived from the single-dish dust continuum data described in the previous section (Sect. 3.1), the virial mass is considerably lower (between factors 2.6 and 7.7, depending on the assumptions for the dust properties and density structure). This implies that the whole region is gravitationally unstable on these scales and likely already undergoing collapse.

Since our PdBI observations are not only sensitive to the continuum but also to the $\mathrm{N}_{2} \mathrm{H}^{+}(1-0)$ emission, we have kinematic information at high spatial resolution as well. Figure 5

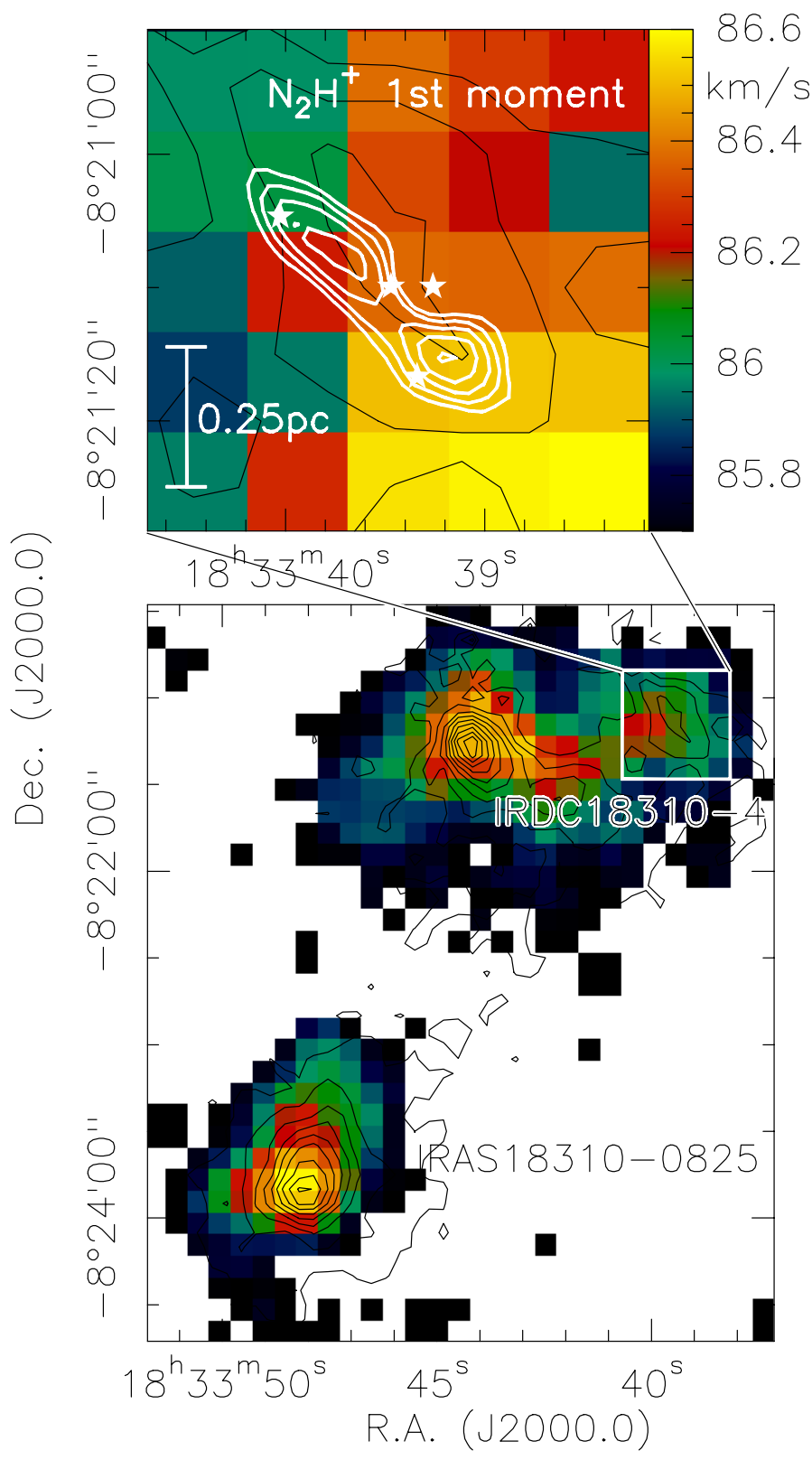

Fig. 4. Bottom panel: integrated $\mathrm{N}_{2} \mathrm{H}^{+}(1-0)$ emission (the whole hyperfine structure between 76 and $94 \mathrm{~km} \mathrm{~s}^{-1}$ ) observed with the Nobeyama $45 \mathrm{~m}$ telescope toward the entire complex containing IRAS 18310-0825 in the south-east and IRDC 18310-4 in the north-west. The contours show the $1.2 \mathrm{~mm}$ continuum data presented first by Beuther et al. (2002). The contouring is from 10 to $90 \%$ of the peak emission of $400 \mathrm{mJy}$ beam $^{-1}$. Top panel: zoom into IRDC $18310-4$ where the color scale presents a 1st moment map (intensity-weighted peak velocity) of the main hyperfine component complex between 84 and $90 \mathrm{~km} \mathrm{~s}^{-1}$. The black contours are the same $1.2 \mathrm{~mm}$ map as in the bottom panel, and the white contours outline the $70 \mu \mathrm{m}$ extinction feature. The stars mark the positions of the four PdBI $3.2 \mathrm{~mm}$ sources.

also shows an $\mathrm{N}_{2} \mathrm{H}^{+}(1-0)$ spectrum extracted toward the peak of mm1 just from the PdBI data (scaled up by a factor 5), and one clearly sees kinematic differences between the large and the small scale. To account for the differences and to complement the PdBI data with the missing flux information, we merged the PdBI and Nobeyama data into one single data-cube (see Sect. 2.4).

Figure 6 presents the spectra extracted from the merged datacube toward the mm peak positions. All these spectra show that 


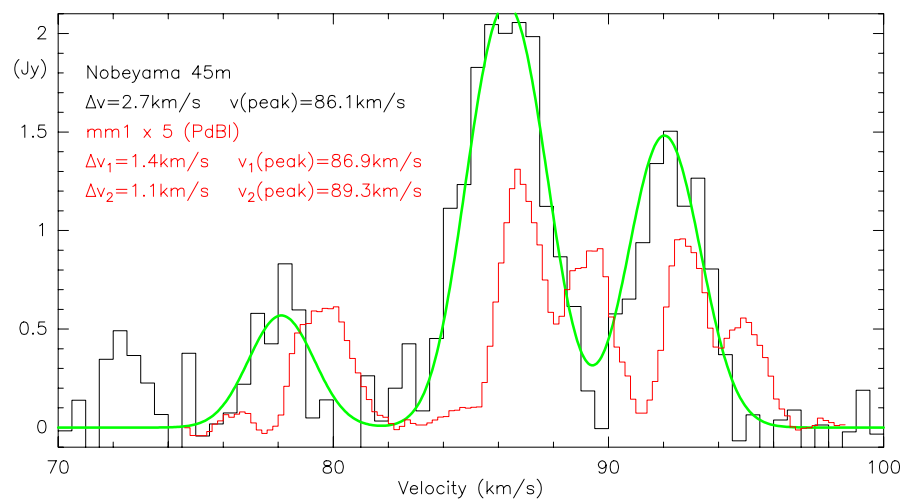

Fig. 5. Compilation of $\mathrm{N}_{2} \mathrm{H}^{+}(1-0)$ Nobeyama $45 \mathrm{~m}$ single-dish spectrum toward IRDC 18310 (black histogram with green fit) and PdBI spectrum toward $\mathrm{mm} 1$ (red histogram). The PdBI data are multiplied by 5 for clarity. Fit results to the whole $\mathrm{N}_{2} \mathrm{H}^{+}(1-0)$ hyperfine structure are given in the panel.

we have at least two separated velocity components in the region, one approximately peaking at $\sim 86.5 \mathrm{~km} \mathrm{~s}^{-1}$ and the other at $\sim 89.3 \mathrm{~km} \mathrm{~s}^{-1}$ (these values are averages from the full hyperfine structure fits to $\mathrm{mm} 1$ to $\mathrm{mm} 3$, see Fig. 6).

The comparison of the PdBI only spectrum and the Nobeyama only spectrum in Fig. 5 shows that the lower-velocity PdBI component corresponds relatively well to the single-dish spectrum whereas the higher-velocity PdBI component does not have a proper single-dish spectrum counterpart. This strongly indicates that the $\sim 86.5 \mathrm{~km} \mathrm{~s}^{-1}$ component is the main component also present in the ambient gas, whereas the higher velocity component at $\sim 89.3 \mathrm{~km} \mathrm{~s}^{-1}$ appears to be mainly associated with a dense sub-part moving with respect to the ambient gas.

To visualize spatial differences between these two spectral components, we imaged the $\mathrm{N}_{2} \mathrm{H}^{+}$data for both velocity structures separately. Figure 7 shows the integrated emission derived for the velocity regimes $[85.5,88.5]$ and $[88.5,89.7] \mathrm{km} \mathrm{s}^{-1}$, respectively. The left panel shows the PdBI- only data whereas the right panel presents the combined PdBI+Nobeyama $45 \mathrm{~m}$ data. To avoid any artifacts of overlapping hyperfine structure lines, we produced these images from only the isolated hyperfine structure component offset by $-8.0 \mathrm{~km} \mathrm{~s}^{-1}(F=0-1)$. While the lower-velocity component agrees well with the mid-infrared absorption structure encompassing $\mathrm{mm} 1, \mathrm{~mm} 3$ and $\mathrm{mm} 4$, the higher-velocity component is peaking mainly in the direction of $\mathrm{mm} 2$. It is at first sight surprising to note that the highervelocity component in the PdBI-only data shows an extension toward $\mathrm{mm} 4$ whereas this is lost in the merged dataset. Most likely this is because that extension is on small scales and comparably weak that it gets lost by the merging process. Additionally, it is possible that the single-dish data add more noise in the combined dataset, which make this weaker extension harder to detect. In addition to this, the merged dataset shows one additional velocity component at even higher velocities between 89.7 and $90.6 \mathrm{~km} \mathrm{~s}^{-1}$ that is more diffusely distributed north of the main IRDC 18310-4 structures. It is unclear whether this is just another unrelated gas component or whether that gas is structurely related to the red-shifted component between 88.5 and $89.7 \mathrm{~km} \mathrm{~s}^{-1}$. Nevertheless, both main velocity components strongly overlap and hence both contribute to the $\mathrm{N}_{2} \mathrm{H}^{+}$emission from $\mathrm{mm} 1$ and $\mathrm{mm} 2$ (see spectra in Fig. 6). Furthermore, the PdBI-only data indicate that $\mathrm{mm} 4$ is located at an interface region between both components. In contrast to this, mm3 appears a bit different because it is located at the edge of the integrated emission of both components.
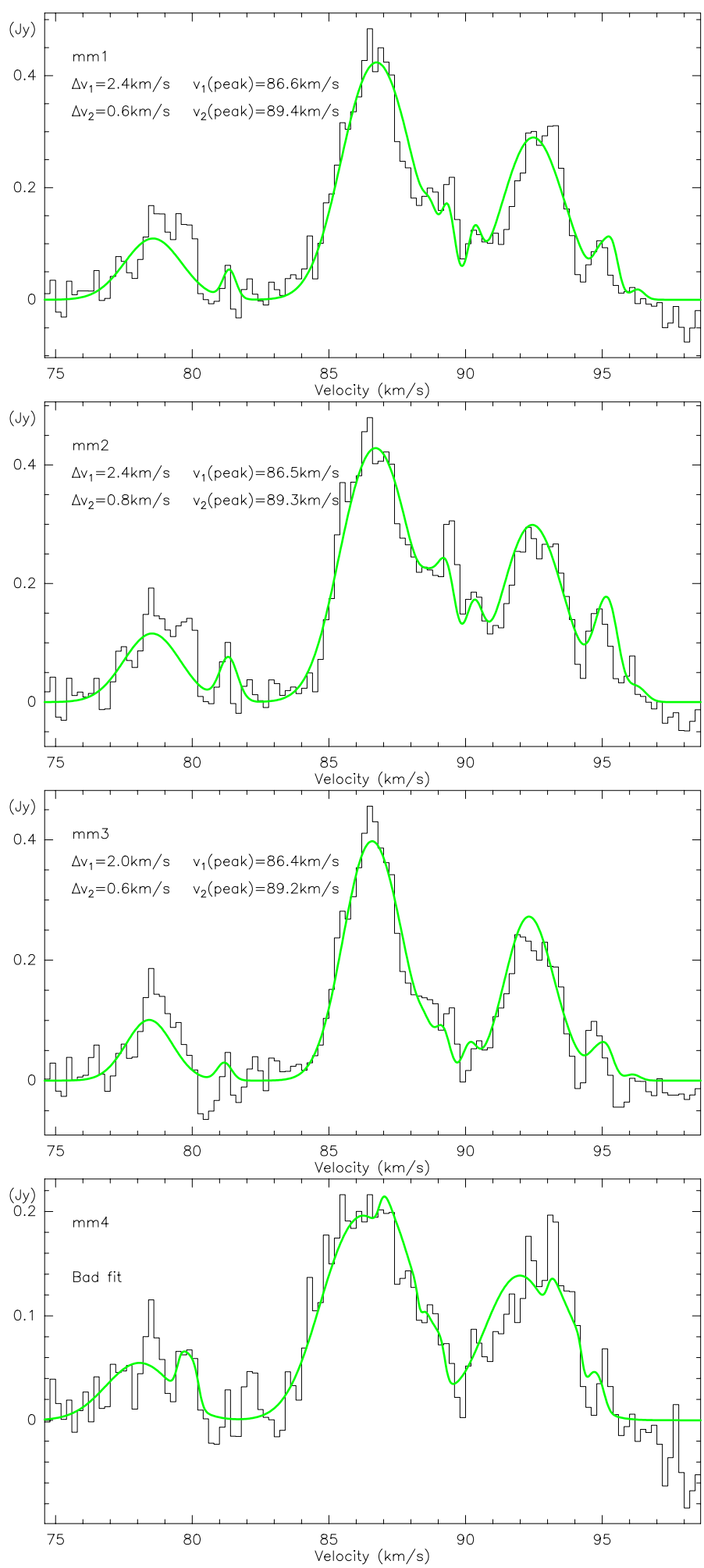

Fig. 6. Spectra and $\mathrm{N}_{2} \mathrm{H}^{+}(1-0)$ hyperfine spectral line fits extracted from the PdBI+Nobeyama $\mathrm{N}_{2} \mathrm{H}^{+}(1-0)$ data toward the mm-peak positions marked in Fig. 2. Fit results and source labels are presented in each panel.

Table 1 lists the line widths of the two components as well as the estimated virial masses following MacLaren et al. (1988) for different density profiles $\left(\rho \propto \frac{1}{r}\right.$ and $\left.\rho \propto \frac{1}{r^{2}}\right)$. The assumed radius of the sources is $1 / 2$ of the FWHM which corresponds at the given distance to $\sim 9000 \mathrm{AU}$. It is obvious that the narrow higher-velocity component barely contributes to the stability 


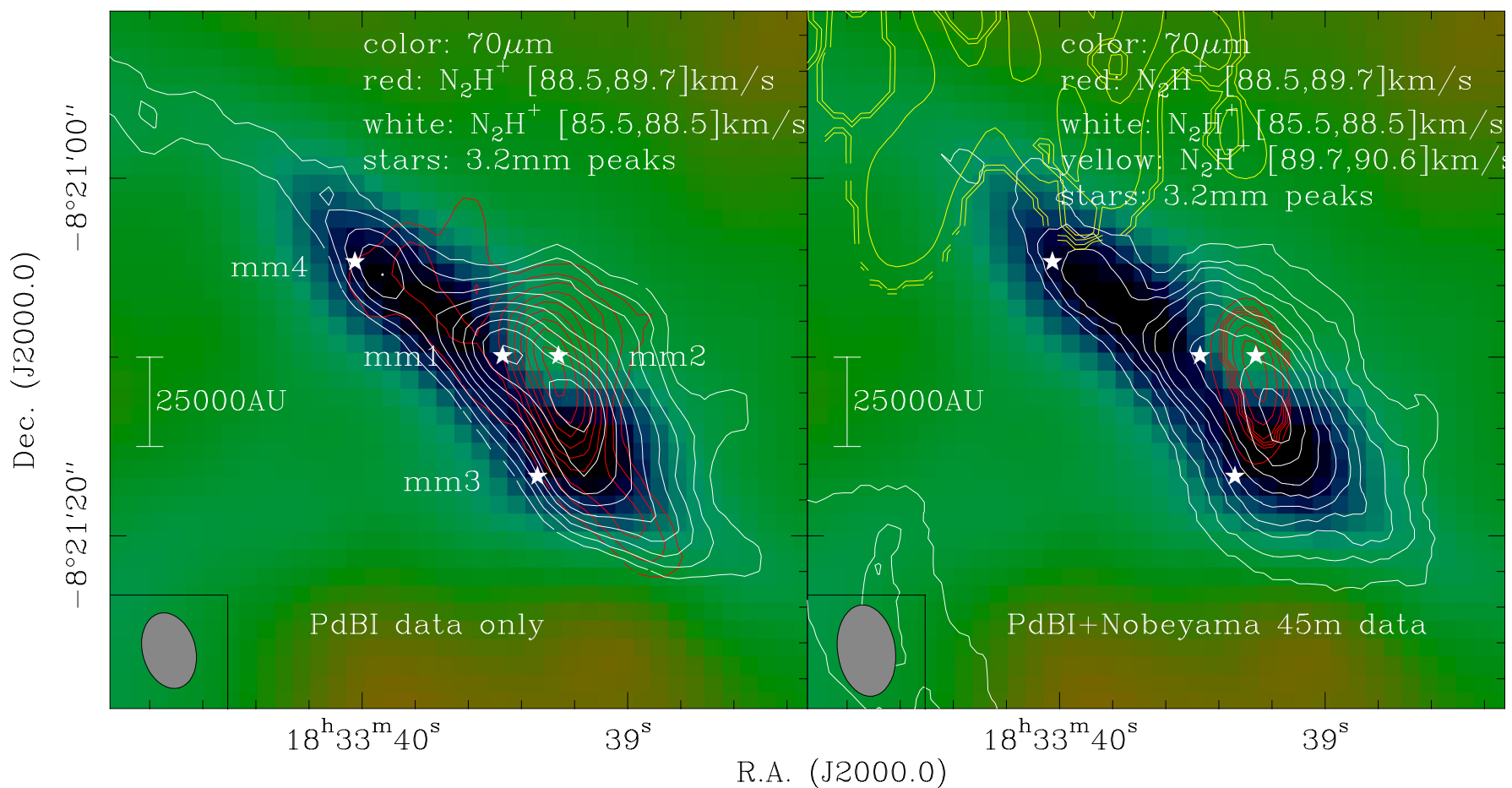

Fig. 7. PdbI only (left panel) and merged PdBI+Nobeyama $45 \mathrm{~m} \mathrm{~N}_{2} \mathrm{H}^{+}(1-0)$ data (right panel). The color-scale shows the $70 \mu \mathrm{m}$ extinction feature, and the contours present the integrated $\mathrm{N}_{2} \mathrm{H}^{+}(1-0)$ emission of three different velocity components as indicated in the figure. To avoid hyperfinesplitting overlap, these integrated maps were created from the isolated hyperfine component $-8.0 \mathrm{~km} \mathrm{~s}^{-1}$ apart from the central component, and then the velocity shifted to the center to be comparable with other line measurements. The contouring is done from 15 to $95 \%$ of the peak emission of each dataset, respectively. The mm-labels, a synthesized beam and a scale-bar are shown as well.

of the cores, and that the broader low-velocity component is far more important to stabilize the structures. Considering the spread in virial masses for different density profiles as well as for the gas masses based on the dust continuum emission and the assumed different dust properties (Table 1), the cores appear closer to virial equilibrium. This is different to the overall virial to gas mass ratio based on the single-dish data for the whole region that is significantly smaller than 1 (see above). However, one has to keep in mind that the virial masses are calculated from the merged $\mathrm{PdBI}+$ Nobeyama $\mathrm{N}_{2} \mathrm{H}^{+}$data whereas the core masses are calculated from the $\mathrm{PdBI}$ data only. If one compares the main component of the $\mathrm{N}_{2} \mathrm{H}^{+}$spectrum toward mm1 without and with the Nobeyama short spacing information (Figs. 5 and 6), one finds that the combined data reveal a FWHM about a factor 1.7 higher than the interferometer data only. Since the line-widths is squared in the estimate of the virial mass, the derived virial masses of the combined data are usually a factor of a few larger than with interferometer data only. This implies that the comparison of masses from the combined PdBI+Nobeyama line data with the PdBI-only continuum data is not reliable. With the missing flux problem, the core masses from the PdBI-only data are underestimated, implying that the ratio of virial to gas mass on the core scales should be smaller than 1 as well. Hence, the cores are also prone to collapse.

Figure 8 presents the integrated $\mathrm{N}_{2} \mathrm{H}^{+}$emission as well as the first and second moment maps (intensity-weighted peak velocities and line widths). In particular the 1st moment map shows that we have no strong velocity gradients across the filament but that the velocity peaks are dominated by the two components discussed above. The 2 nd moment map appears as if it had a line width increase toward $\mathrm{mm} 2$, however that is only because the 2nd moment algorithm assumes a single component, and therefore the two real components appear as one broader feature in that direction.

\section{Discussion}

\subsection{Linear scales and densities from low- to high-mass starless gas clumps}

How do linear scales and average densities compare between star-forming regions from low- to high-mass? To compare the different mass regimes with the high-mass starless region presented in this paper, we selected the low-mass starless core B68 (e.g., Alves et al. 2001; Nielbock et al. 2012) which resembles typical other low-mass globules (e.g., Launhardt et al. 2010), and the intermediate-mass starless star-forming region IRDC 19175 (Beuther \& Henning 2009). Figure 9 presents data for all three regions. It is interesting to note that all three regions have approximately the same linear spatial extent on the order of $50000 \mathrm{AU}$. However, while the linear size between the three regions does not vary considerably, the total gas reservoirs for the three complexes are very different, ranging from $\sim 3.1 M_{\odot}$ for the low-mass core B68 (Nielbock et al. 2012), $\sim 87 M_{\odot}$ for the intermediate-mass region IRDC 19175 (Beuther \& Henning 2009 ) to values between $\sim 800$ and $\sim 1600 M_{\odot}$ (depending on the dust model, see Sect. 3.1) for the high-mass region IRDC 183104 (see Table 3 for a list of the source parameters). Assuming that the three regions resemble typical starless gas clumps in the different mass regimes, these data imply that not the linear sizes are important but the mass reservoir squeezed within such regions, i.e., the densities.

While the peak density of the B68 core is $\sim 3.4 \times 10^{5} \mathrm{~cm}^{-3}$ (Nielbock et al. 2012), the average density of the region is considerably lower. We estimate an average density for B 68 from the total mass of $3.1 M_{\odot}$ (recent analysis of Herschel data, Nielbock et al. 2012) divided by a sphere with approximate radius of $\sim 150^{\prime \prime}$ at a given distance of $\sim 150 \mathrm{pc}$. Using these numbers the average gas density of the low-mass starless core B68 is 


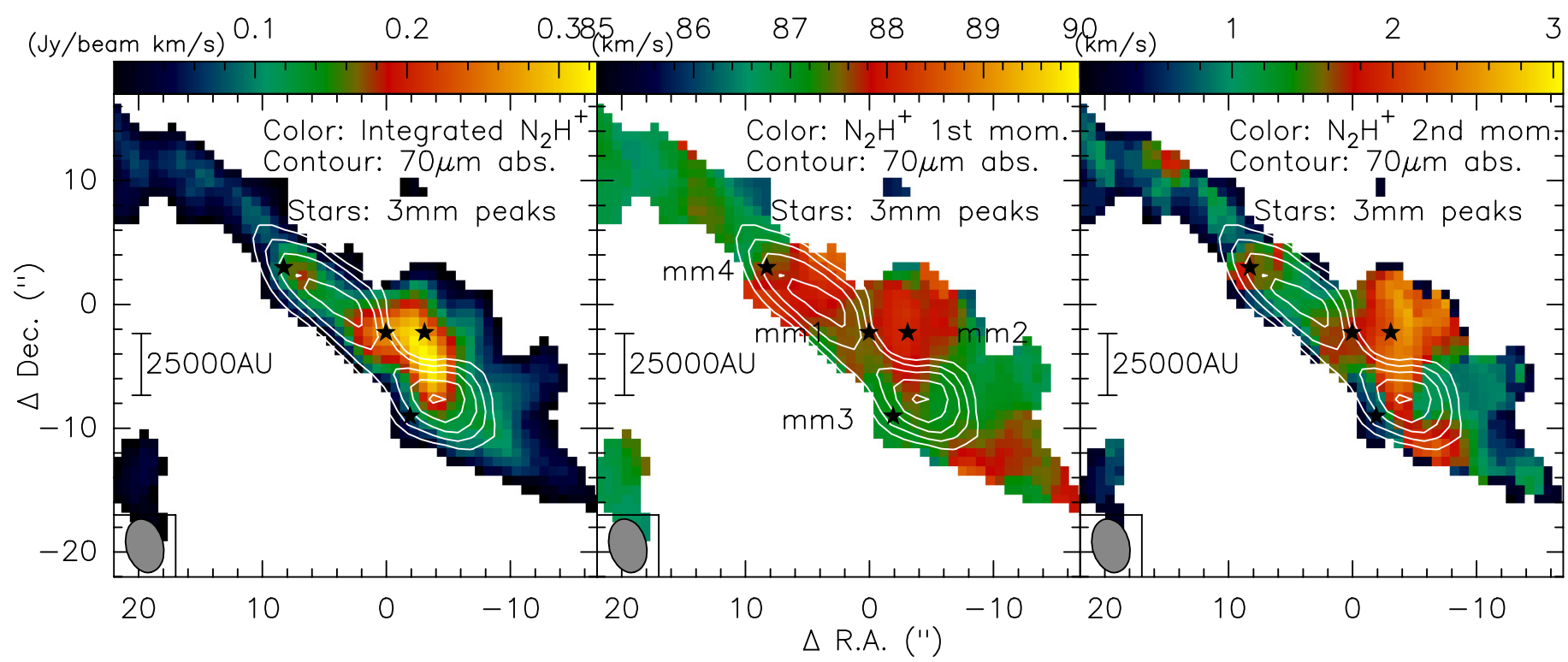

Fig. 8. $\mathrm{N}_{2} \mathrm{H}^{+}(1-0)$ moment maps of the PdBI-only data toward IRDC 18310-4. The left, middle and right panel show in color the 0th moment (integrated intensity), 1st moment (intensity-weighted peak velocity) and 2nd moment (intensity-weighted linewidth), respectively. The moments were again extracted for the isolated hyperfine component $-8.0 \mathrm{~km} \mathrm{~s}^{-1}$ from the line center. The $1 \mathrm{st}$ moment was calculated from this isolated hyperfine component and then shifted toward by $+8.0 \mathrm{~km} \mathrm{~s}^{-1}$ to the $v_{\mathrm{lsr}}$ of the source again. The white contours outline the $70 \mu \mathrm{m}$ extinction feature and the stars mark the $3.2 \mathrm{~mm}$ peak positions from Fig. 2. The synthesized beam and a scale-bar are shown again.
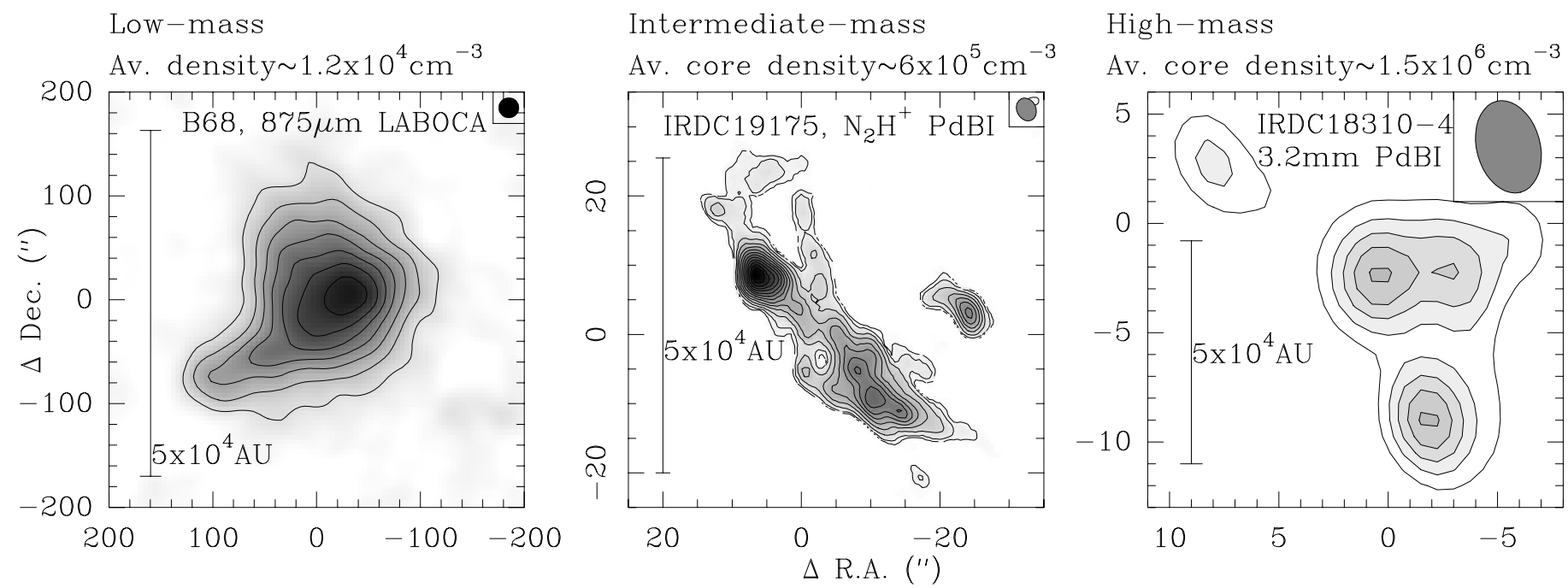

Fig. 9. Comparison between starless gas-clumps from low- via intermediate- to high-mass star formation. The $875 \mu \mathrm{m}$ data for the prototypical low-mass core B68 are taken from Nielbock et al. (2012), and the $\mathrm{N}_{2} \mathrm{H}^{+}(1-0)$ data for the intermediate-mass star-forming region IRDC 19175 are from Beuther \& Henning (2009). Each panel presents a linear scale-bar of $50000 \mathrm{AU}$ and the synthesized beam, average densities are mentioned as well.

approximately $1.2 \times 10^{4} \mathrm{~cm}^{-3}$ over a region corresponding to a sphere with $\sim 45000 \mathrm{AU}$ diameter.

For the intermediate-mass star-forming region IRDC 19175, average core densities are estimated by Beuther \& Henning (2009) to $\sim 6 \times 10^{5} \mathrm{~cm}^{-3}$ over regions corresponding to spheres with $\sim 3500 \mathrm{AU}$ diameter. Estimating an average density from the total mass of $87 M_{\odot}$ over a volume corresponding to an effective radius of the $1.2 \mathrm{~mm}$ continuum map in Beuther \& Henning (2009) results only in slightly lower values around $\sim 2.6 \times 10^{5} \mathrm{~cm}^{-3}$ over a region corresponding to a sphere with $\sim 48000$ AU diameter.

To compare these values with our high-mass star-forming region IRDC 18310-4, we estimate an average density for the core $\mathrm{mm} 1$ via averaging the peak column densities listed in Table 1 for the two different dust models, and assuming that the 3rd dimension has the same size as our synthesized beam. This results in average densities for mm1 of $\sim 1.5 \times 10^{6} \mathrm{~cm}^{-3}$ over a region corresponding to a sphere with $\sim 18000 \mathrm{AU}$ diameter. To get the density over a slightly larger regions, we use the average column density derived in Sect. 3.1 for the two different dust models from the single-dish $1.2 \mathrm{~mm}$ continuum data and again assume the 3rd dimension to have the same spatial extent as the single-dish beam of 10.5". This results in average densities of $\sim 2.5 \times 10^{5} \mathrm{~cm}^{-3}$ over a region corresponding to a sphere with $\sim 51000$ AU diameter.

These large density differences obviously also have a strong impact on the fragmentation properties of the starless gas clumps. While the low-density low-mass core B68 does not fragment at all, the higher-density intermediate- to high-mass starless clumps do fragment significantly. To better understand these 
Table 3. Star-forming region parameters.

\begin{tabular}{|c|c|c|c|}
\hline & B68 & IRDC19175 & IRDC18310-4 \\
\hline Mass $\left[M_{\odot}\right]$ & 3.1 & 87 & $800-1600$ \\
\hline Av. dens. $\left[\mathrm{cm}^{-3}\right]$ & $1.2 \times 10^{4}$ & $2.6 \times 10^{5}$ & $2.5 \times 10^{5}$ \\
\hline Size [AU] & 45000 & 48000 & 51000 \\
\hline Jeans length $^{2}[\mathrm{AU}]$ & 44000 & 10000 & 10000 \\
\hline Jeans mass ${ }^{2}\left[M_{\odot}\right]$ & 1.7 & 0.36 & 0.37 \\
\hline Core dens. $\left[\mathrm{cm}^{-3}\right]$ & -1 & $6.0 \times 10^{5}$ & $1.5 \times 10^{6}$ \\
\hline Jeans length $^{2}$ [AU] & $-^{1}$ & 6000 & 4000 \\
\hline Jeans mass ${ }^{2}\left[M_{\odot}\right]$ & $-{ }^{1}$ & 0.24 & 0.15 \\
\hline
\end{tabular}

Notes. ${ }^{(1)}$ B68 as a whole is already the core. ${ }^{(2)}$ At $15 \mathrm{~K}$.

properties, we estimate the Jeans length for the average B68 density of $1.2 \times 10^{4} \mathrm{~cm}^{-3}$, the approximate average large-scale densities of IRDC 19175 and IRDC $18310-4$ of $\sim 2.5 \times 10^{5} \mathrm{~cm}^{-3}$, and the higher core densities of the two regions of $\sim 6 \times 10^{5} \mathrm{~cm}^{-3}$ and $\sim 1.5 \times 10^{6} \mathrm{~cm}^{-3}$, respectively. Assuming average temperatures of $15 \mathrm{~K}$ for all these starless regions, the corresponding Jeans-length are 44000 AU (B68), 10000 AU (average largescale densities of IRDC 19175 \& IRDC 18310-4), 6000 AU (average core density of IRDC 19175) and $\sim 4000 \mathrm{AU}$ (average core density of IRDC 18310-4), respectively. This relatively simple isothermal Jeans-analysis already explains the observations well: The large Jeans-length for B68 is reflected in the fact that this low-mass starless core does not fragment on the given scales (Fig. 9). Furthermore, as already discussed in Beuther \& Henning (2009), the different Jeans-lengths for B68 (located close to the Pipe nebula) and IRDC 19175 correspond well to the observed core separations in the low-mass Pipe nebula and the intermediate-mass IRDC 19175 region, respectively. For the high-mass region IRDC 18310-4, we are still spatial resolution limited and only observe the fragmentation on the scales of our observed resolution elements (see also Table 2). In future higherspatial-resolution observations, we expect to resolve this region into even more and closer spaced cores.

Calculating the Jeans-mass instead of the Jeans-length for the average densities of B68, IRDC 19175 and IRDC 183104, we find $1.7,0.36$ and $0.37 M_{\odot}$, respectively. The Jeansmasses for the higher core densities are correspondingly smaller (Table 3). For the low-mass region B68 that value is on the order of the whole mass $\left(3.1 M_{\odot}\right)$, and also for the intermediate-mass region IRDC 19175, the estimate masses of the individual cores are mostly below $1 M_{\odot}$. Hence Jeans-masses and core masses approximately agree in these two cases. In contrast to that, the Jeans-mass in IRDC 18310-4 is significantly lower than the mass estimates we derived from the dust continuum data (Table 1). Therefore, similar as discussed in the previous paragraph, for the high-mass region IRDC 18310-4, we are still spatial resolution limited, and it is highly likely that the here presented cores $\mathrm{mm} 1$ to $\mathrm{mm} 4$ will fragment even further when observed at higher spatial resolution.

Assuming that these example regions resemble starless gas clumps over the whole mass regime, one may conclude that gravitational instability in the classical Jeans-analysis explains the fragmentation properties of starless gas clumps from low- to high-mass reasonably well. The major difference between the regions appears to be their densities and mass reservoirs squeezed into the respective spatial scales. Similar Jeans fragmentation scales have also been observed toward more evolved regions, e.g., the young cluster NGC 2264 (Teixeira et al. 2006). In contrast to the larger-scale cloud fragmentation where turbulence plays a major role (e.g., Padoan \& Nordlund 2002; Mac Low \& Klessen 2004), these data indicate that the fragmentation on smaller clump and core scales appears to be dominated by gravity.

\subsection{Kinematic signatures of a dynamic cloud collapse}

Independent of the cloud formation models - do clouds evolve slower via ambipolar diffusion processes (e.g., Mouschovias et al. 2006; McKee \& Ostriker 2007) or faster via more dynamic gas flows (e.g., Vázquez-Semadeni et al. 2006; Heitsch et al. 2008; Banerjee et al. 2009) - the gas has to show dynamic signatures of contraction and/or infall over large spatial scales. During molecular cloud formation the diffuse HI gas converts to the denser molecular phase. Within molecular clouds, filamentary sub-structures are likely important, and recent data indicate that hierarchical, often also filamentary structures feed the central and densest filaments or gas clumps (e.g., Myers 2009; Schneider et al. 2010; Schmalzl et al. 2010; Hennemann et al. 2012). These central cluster-forming clumps have then to collapse and form the multiple and clustered stellar associations in the end. Statistical signatures of infall from single-dish average line profiles exist. However, they are often ambiguous, and one finds signatures for infall as well outflowing motions (e.g., Fuller et al. 2005). More coherent infall motions on larger scales have only rarely been reported (e.g., Motte et al. 2005; Schneider et al. 2010). Going from the cluster-forming scales down to cores forming individual or multiple, bound objects, dynamic studies in the past have mostly concentrated on more evolved evolutionary stages like high-mass protostellar objects (HMPOs) or ultracompact HII regions (e.g., Keto 2002; Sollins et al. 2005; Sandell et al. 2009; Qiu et al. 2011; Goddi et al. 2011; Beuther et al. 2012a).

While the above infall studies dealt with the average infall motions of the entire star-forming gas clumps, only recently spatially resolved dynamical studies of sub-structures and cores within the natal gas became possible (e.g., Beuther \& Henning 2009; Zhang \& Wang 2011; Wang et al. 2011; Csengeri et al. $2011 \mathrm{a}, \mathrm{b})$. In contrast to the above discussed single-dish studies where often infall signatures are derived from line profiles of optically thick lines, Ragan et al. (2012b) also find signatures of collapse and fragmentation via high-spatial-resolution $\mathrm{NH}_{3}$ observations of IRDCs. Furthermore, the studies by Csengeri et al. $(2011 a, b)$ revealed multiple velocity components in optically thin gas tracers like $\mathrm{H}^{13} \mathrm{CO}^{+}$or $\mathrm{N}_{2} \mathrm{H}^{+}$that were interpreted as signatures of shear motions possibly caused by converging gas flows.

The spectra we found (Figs. 5 and 6) also show multiple velocity components in the dense gas tracer $\mathrm{N}_{2} \mathrm{H}^{+}$. Figure 7 even identifies that the different velocity components are dominated by different $\mathrm{mm}$ cores within the large-scale gas clump. In addition to the work by Csengeri et al. (2011a,b), multiple velocity components in optically thin dense gas tracers were also found by Beuther \& Henning (2009) as well as in recent work by Ragan et al. (in prep.) with the PdBI toward the G11.11 filament, and by Bihr et al. (submitted) with VLA $\mathrm{NH}_{3}$ observations toward IRDCs. Combining our new data with the results recently found by the other groups, based on high-spatial resolution interferometer observations there emerges a regular signature of multiple velocity components in optically thin dense gas tracers. Typical separations line widths of these multiple velocity components are around $\sim 2 \mathrm{~km} \mathrm{~s}^{-1}$ and $\sim 1 \mathrm{~km} \mathrm{~s}^{-1}$, respectively.

Confronting these observational results with theory, Rowan Smith has recently started to simulate the spectral line emission 


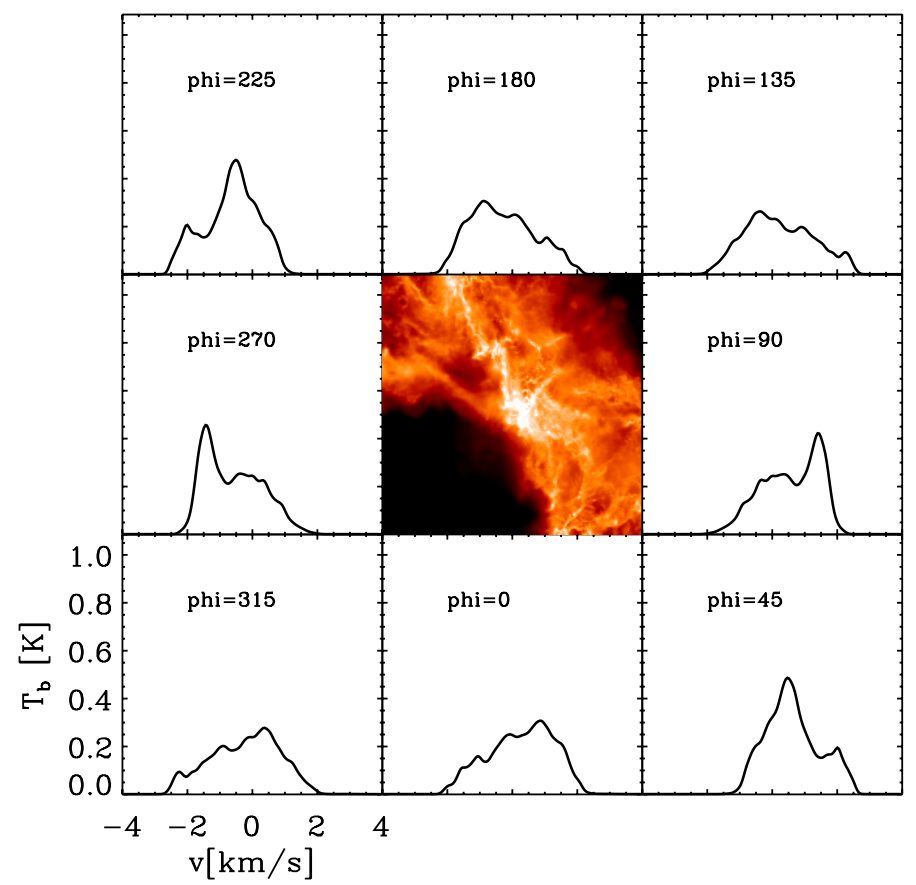

Fig. 10. $\mathrm{N}_{2} \mathrm{H}^{+}(1-0)$ isolated hyperfine line profiles viewed through various lines of sight through a massive star forming region. The central image with a side-length of $0.8 \mathrm{pc}$ shows the column density of the region and the surrounding line profiles are calculated for a $0.09 \mathrm{pc}$ beam centered directly on the embedded core and viewed along the direction each panel touches the central image.

from dynamically collapsing clumps of a large-scale collapsing gas cloud modeled initially by Smith et al. (2009) and Bonnell et al. (2011). First results about cores associated with low-mass star formation were presented in Smith et al. (2012), and the simulations for high-mass collapsing gas clumps are currently conducted (Smith et al. 2013). Figure 10 shows an example of non-Gaussian optically thin line profiles produced from a simulation of a massive star-forming gas clump with $370 M_{\odot}$ (Smith et al. 2009, 2013). The $\mathrm{N}_{2} \mathrm{H}^{+}(1-0)$ line profiles were calculated using an assumed abundance ratio of $10^{-10}$ relative to molecular hydrogen (Aikawa et al. 2005). The radiative transfer code RADMC-3D (Dullemond 2012) was used to calculate the line emission given the temperatures and densities from the original simulation. (For a full description of our method see Smith et al. 2012). Here, only the isolated hyperfine component $F=0-1$ (see Fig. 6) offset by $\sim 8.0 \mathrm{~km} \mathrm{~s}^{-1}$ from the central component is shown. The line profiles are synthesized over a beam of FWHM $0.09 \mathrm{pc}$ and calculated for a variety of viewing angles. No artificial noise is added to the data.

Clearly, these simulations find similar signatures of multiple $\mathrm{N}_{2} \mathrm{H}^{+}$components within the dynamically collapsing gas clump. Typical peak separations and line widths are similar to what is found in the observational data. The multiple components seen in Fig. 10 arise in the simulations due to the clumpy nature of the high-mass star-forming region. Across the region there is a large scale supersonic collapse motion running from around $+2 \mathrm{~km} \mathrm{~s}^{-1}$ on one side to $-2 \mathrm{~km} \mathrm{~s}^{-1}$ on the other. Dense clumps of gas at different positions along this velocity gradient all contribute to the total emission and produce peaks in the line profile at their individual velocities. A full analysis of line profiles from massive star formations will be presented in Smith et al. (submitted).

This interpretation is also consistent with our finding that the ratio of viral mass to total gas mass is significantly smaller than 1 for the entire region, implying that the large-scale gas clump is prone to collapse, similar to the findings of Ragan et al. (2012b) for their sample of IRDCs. Since the virial mass to core mass ratio is smaller than 1 on the core scales as well, the region is consistent with a globally collapsing gas clumps on almost all scales.

We can also estimate the dynamical crossing time as well as the free-fall time of the region. Using the typical velocity difference between the two gas components of $\sim 2.8 \mathrm{~km} \mathrm{~s}^{-1}$ as the velocity dispersion of the clumps, and the approximate extent of the $70 \mu \mathrm{m}$ absorption feature of $\sim 30^{\prime \prime}$ (corresponding to $147000 \mathrm{AU}$ at the given distance), the dynamical crossing time for IRDC $18310-4$ is $\sim 2.5 \times 10^{5}$ yrs. In comparison to that, estimating the free-fall time for a $1000 M_{\odot}$ gas clump of with a radius of half the previously used extend, we get an approximate free-fall time-scale of $\sim 1.2 \times 10^{5} \mathrm{yrs}$. Although there is a difference of about a factor 2 between the two estimates, these are just approximate values and they agree order-of-magnitude wise. This again implies that a dynamical collapse of the whole gas clump is a feasible model for this region.

Combining our observational results with the modeling of line profiles for dynamically collapsing gas clumps, we identify signatures that even this very young, still starless high-mass gas clump is actively collapsing and thus at the verge of star formation. The fact that similar signatures were recently found toward other regions as well, indicates that multiple velocity components of optically thin gas tracers are an excellent tool to identify and study the earliest stages of dynamical infall in high-mass star formation.

\section{Conclusions}

Since the evolutionary time-scale for high-mass starless gas clumps is extremely short (e.g., Motte et al. 2007; Russeil et al. 2010; Tackenberg et al. 2012), so far they had barely been studied in great depth at high spatial resolution (some notable recent papers in that field are Csengeri et al. 2011a; Ragan et al. 2012b). To better understand the fragmentation and dynamical properties of the most massive gas clumps during their earliest evolutionary stages, here we present an interferometric PdBI kinematic and continuum study of a prototypical high-mass starless gas clump that remains far-infrared dark up to $100 \mu \mathrm{m}$ wavelengths.

The gas reservoir between $\sim 800$ and $\sim 1600 M_{\odot}$ (depending on the assumed dust properties) splits up at a spatial resolution of $4.3^{\prime \prime} \times 3.0^{\prime \prime}$ (linear scales $21000 \times 15000 \mathrm{AU}$ ) into 4 cores. A comparison of this high-mass starless region with typical lowmass (B68) and intermediate-mass (IRDC 19175) star-forming forming regions reveals that the overall spatial size of the lowmass to high-mass star-forming regions does not vary significantly. While the linear extent of the regions is similar, the mass reservoir squeezed into this spatial size on the order of $0.25 \mathrm{pc}$, and hence the densities do vary by about 2 orders of magnitude. A classical gravitational instability Jeans analysis is capable to explain the fragmentation properties of the gas clumps over the whole mass range presented.

In addition to the fragmentation properties, we find multiple spectral velocity components toward the resolved gas cores. Recent radiative transfer hydrodynamic simulations of dynamical collapsing high-mass gas clumps are consistent with these multiple velocity components. This also agrees with a ratio between estimated viral and total mass of the whole region $<1$.

In summary, although this massive gas clump does not have any embedded protostellar source down to our Herschel far-infrared detection limits, the fragmentation and dynamical 
properties of the gas and dust are consistent with early collapse motion and clustered star formation.

Acknowledgements. We like to thank Joao Alves for interesting discussions about the comparison between high- and low-mass star-forming regions Furthermore, thanks a lot to a careful referee who helped improving the paper.

\section{References}

Aikawa, Y., Herbst, E., Roberts, H., \& Caselli, P. 2005, ApJ, 620, 330

Alves, J. F., Lada, C. J., \& Lada, E. A. 2001, Nature, 409, 159

Alves, J., Lombardi, M., \& Lada, C. J. 2007, A\&A, 462, L17

Banerjee, R., Vázquez-Semadeni, E., Hennebelle, P., \& Klessen, R. S. 2009, MNRAS, 398, 1082

Bergin, E. A., Hartmann, L. W., Raymond, J. C., \& Ballesteros-Paredes, J. 2004, ApJ, 612, 921

Beuther, H., \& Henning, T. 2009, A\&A, 503, 859

Beuther, H., Schilke, P., Menten, K. M., et al. 2002, ApJ, 566, 945

Beuther, H., Churchwell, E. B., McKee, C. F., \& Tan, J. C. 2007, in Protostars and Planets V, eds. B. Reipurth, D. Jewitt, \& K. Keil, 165

Beuther, H., Henning, T., Linz, H., et al. 2010, A\&A, 518, L78

Beuther, H., Linz, H., \& Henning, T. 2012a, A\&A, 543, A88

Beuther, H., Tackenberg, J., Linz, H., et al. 2012b, A\&A, 538, A11

Bonnell, I. A., Smith, R. J., Clark, P. C., \& Bate, M. R. 2011, MNRAS, 410, 2339

Bontemps, S., Motte, F., Csengeri, T., \& Schneider, N. 2010, A\&A, 524, A18

Carey, S. J., Noriega-Crespo, A., Mizuno, D. R., et al. 2009, PASP, 121, 76

Commerçon, B., Hennebelle, P., \& Henning, T. 2011, ApJ, 742, L9

Csengeri, T., Bontemps, S., Schneider, N., Motte, F., \& Dib, S. 2011a, A\&A, 527, A135

Csengeri, T., Bontemps, S., Schneider, N., et al. 2011b, ApJ, 740, L5

Draine, B. T., Dale, D. A., Bendo, G., et al. 2007, ApJ, 663, 866

Dullemond, C. P. 2012, Astrophysics Source Code Library, 2015

Fuller, G. A., Williams, S. J., \& Sridharan, T. K. 2005, A\&A, 442, 949

Goddi, C., Moscadelli, L., \& Sanna, A. 2011, A\&A, 535, L8

Griffin, M. J., Abergel, A., Abreu, A., et al. 2010, A\&A, 518, L3

Heitsch, F., Hartmann, L. W., Slyz, A. D., Devriendt, J. E. G., \& Burkert, A. 2008, ApJ, 674, 316

Hennemann, M., Motte, F., Schneider, N., et al. 2012, A\&A, 543, L3

Henning, T., Linz, H., Krause, O., et al. 2010, A\&A, 518, L95

Jenkins, E. B. 2004, in Origin and Evolution of the Elements, eds. A. McWilliam,

\& M. Rauch, 336

Keto, E. 2002, ApJ, 568, 754

Kroupa, P. 2001, MNRAS, 322, 231

Krumholz, M. R., \& McKee, C. F. 2008, Nature, 451, 1082

Launhardt, R., Nutter, D., Ward-Thompson, D., et al. 2010, ApJS, 188, 139
Launhardt, R., Stutz, A. M., Schmiedeke, A., et al. 2013, A\&A, 551, A98

Linz, H., Krause, O., Beuther, H., et al. 2010, A\&A, 518, L123

Mac Low, M., \& Klessen, R. S. 2004, Rev. Mod. Phys., 76, 125

MacLaren, I., Richardson, K. M., \& Wolfendale, A. W. 1988, ApJ, 333, 821

Mathis, J. S., Rumpl, W., \& Nordsieck, K. H. 1977, ApJ, 217, 425

McKee, C. F., \& Ostriker, E. C. 2007, ARA\&A, 45, 565

Motte, F., Bontemps, S., Schilke, P., et al. 2005, in Massive Star Birth: A Crossroads of Astrophysics, eds. R. Cesaroni, M. Felli, E. Churchwell, \& M. Walmsley, IAU Symp., 227, 151

Motte, F., Bontemps, S., Schilke, P., et al. 2007, A\&A, 476, 1243

Mouschovias, T. C., Tassis, K., \& Kunz, M. W. 2006, ApJ, 646, 1043

Myers, P. C. 2009, ApJ, 700, 1609

Nielbock, M., Launhardt, R., Steinacker, J., et al. 2012, A\&A, 547, A11

Ossenkopf, V., \& Henning, T. 1994, A\&A, 291, 943

Ott, S. 2010, in Astronomical Data Analysis Software and Systems XIX, eds. Y. Mizumoto, K.-I. Morita, \& M. Ohishi, ASP Conf. Ser., 434, 139

Padoan, P., \& Nordlund, ̊.. 2002, ApJ, 576, 870

Pilbratt, G. L., Riedinger, J. R., Passvogel, T., et al. 2010, A\&A, 518, L1

Poglitsch, A., Waelkens, C., Geis, N., et al. 2010, A\&A, 518, L2

Qiu, K., Zhang, Q., \& Menten, K. M. 2011, ApJ, 728, 6

Ragan, S., Henning, T., Krause, O., et al. 2012a, A\&A, 547, A49

Ragan, S. E., Heitsch, F., Bergin, E. A., \& Wilner, D. 2012b, ApJ, 746, 174

Reid, M. J., Menten, K. M., Zheng, X. W., et al. 2009, ApJ, 700, 137

Roussel, H. 2012 [arXiv: 1205.2576]

Russeil, D., Zavagno, A., Motte, F., et al. 2010, A\&A, 515, A55

Sandell, G., Goss, W. M., Wright, M., \& Corder, S. 2009, ApJ, 699, L31

Sawada, T., Ikeda, N., Sunada, K., et al. 2008, PASJ, 60, 445

Schmalzl, M., Kainulainen, J., Quanz, S. P., et al. 2010, ApJ, 725, 1327

Schneider, N., Csengeri, T., Bontemps, S., et al. 2010, A\&A, 520, A49

Schuller, F., Menten, K. M., Contreras, Y., et al. 2009, A\&A, 504, 415

Smith, R. J., Clark, P. C., \& Bonnell, I. A. 2009, MNRAS, 396, 830

Smith, R. J., Shetty, R., Stutz, A. M., \& Klessen, R. S. 2012, ApJ, 750, 64

Smith, R. J., Shetty, R., Beuther, H., Klessen, R. S., \& Bonnell, I. A. 2013, ApJ, accepted [arXiv: 1304.4950$]$

Sollins, P. K., Zhang, Q., Keto, E., \& Ho, P. T. P. 2005, ApJ, 624, L49

Sridharan, T. K., Beuther, H., Schilke, P., Menten, K. M., \& Wyrowski, F. 2002, ApJ, 566, 931

Sridharan, T. K., Beuther, H., Saito, M., Wyrowski, F., \& Schilke, P. 2005, ApJ, 634, L57

Stutz, A., Launhardt, R., Linz, H., et al. 2010, A\&A, 518, L87

Tackenberg, J., Beuther, H., Henning, T., et al. 2012, A\&A, 540, A113

Tackenberg, J., Beuther, H., Henning, Th., et al. 2013, A\&A, submitted

Teixeira, P. S., Lada, C. J., Young, E. T., et al. 2006, ApJ, 636, L45

Vázquez-Semadeni, E., Ryu, D., Passot, T., González, R. F., \& Gazol, A. 2006, ApJ, 643, 245

Wang, K., Zhang, Q., Wu, Y., \& Zhang, H. 2011, ApJ, 735, 64

Zhang, Q., \& Wang, K. 2011, ApJ, 733, 26

Zinnecker, H., \& Yorke, H. W. 2007, ARA\&A, 45, 481 